\title{
Intrinsic and Extrinsic Stability of Ovonic-Switching Devices
}

\author{
F. Buscemi, ${ }^{1}$ E. Piccinini, R. Brunetti, ${ }^{1}$ M. Rudan \\ "E. De Castro" Advanced Research Center on Electronic Systems (ARCES) and Department DEI \\ University of Bologna, Viale Risorgimento 2, I-40136 Bologna, Italy — mrudan@arces.unibo.it \\ ${ }^{1}$ Department of Physics, Informatics, and Mathematics \\ University of Modena and Reggio Emilia, Via Campi 213/A, I-41125, Modena, Italy
}

\begin{abstract}
The time evolution of current and voltage in Ovonicswitching devices is affected, on one side, by parasitic elements due to contacts and connectors and, on the other one, by the internal-relaxation mechanisms of the material itself. The two aspects, respectively termed here "intrinsic" and "extrinsic" dynamics, are investigated in this paper on the basis of the time-dependent, trap-limited conduction model proposed by the authors for investigating this type of devices.
\end{abstract}

\section{Introduction}

The electrical switching observed in a class of materials, referred to as "Ovonic", is due to a transition process from a high- to a low-resistivity state occurring when a threshold field is reached [1]- [15]. The investigations on these materials' models have strongly been stimulated by the technological exploitation in the design of phasechange memories [12]. Such applications require not only predictive tools for the threshold switching in steady-state conditions, but also the analysis of the electric behavior of Ovonic materials in the dynamic and transient regime. The $I(V)$ characteristic of Ovonic materials of interest shows a negative differential-resistance region above the threshold point, which may give rise to instability. Besides degrading the quality of the $I(V)$-curve measurement (due to instability, in fact, only the positive-slope branches may actually be traced), instability may hamper the functioning of the selectors, made of Ovonic materials, used in advanced memory architectures [16]. There are at least two causes of instability: $i$ ) the unavoidable presence of reactive parasitic elements due to contacts and connectors and, ii) the internal-relaxation mechanisms of the material itself. The first type of parasitic elements may be approximated with lumped linear capacitors or inductors [17], [18] like, e.g., in Fig. 1 (specifically, $R_{S}$ and $C$ are parasitic elements of the Ovonic material, while $R_{L}$ belongs to the measuring equipment); thus, their incorporation into the device model is achieved without changing the standard description based on voltages, currents, and their derivatives. In contrast, the internal-relaxation mechanisms are not necessarily amenable to a description based on the standard variables, this making the analysis more involved. This paper addresses the issue of a global model of an Ovonic device based on the lumped-element structure, investigates the origin of a possible oscillatory behavior due to instabilities, and identifies the corresponding relaxation times. The work is based on the analytical model of Ovonic switching presented in [19], relying on a thermally-assisted, trap-limited conduction scheme, which has been shown able to reproduce electrical bistability and has more recently been extended to the dynamic case [20].

\section{Model}

The starting point is the time-dependent model of [20]:

$$
\begin{gathered}
J=q \mu n_{B} F, \quad \frac{n}{n_{B}}=1+c \exp \left(\frac{\Delta E}{k T_{e}}\right), \\
J F=n k \frac{T_{e}-T_{0}}{\tau_{R}}+\Delta E \frac{\mathrm{d}}{\mathrm{d} t}\left[n_{B}\left(T_{e}\right)-n_{B}\left(T_{0}\right)\right],
\end{gathered}
$$

where $J, q, \mu$, and $F$ are the current density, electronic charge, mobility of the band electrons, and electric field, respectively. As the sample under investigation is assumed one dimensional and uniform, the equations bear no dependence on position. In turn, $n, n_{B}$ are the concentrations of all electrons and of band electrons, respectively, while $k, T_{e}, T_{0}$, and $\tau_{R}$ are the Boltzmann constant, the temperature of the band electrons, the room temperature, and the electron-temperature relaxation time. Finally, $\Delta E$ is the difference between the energy of the band and that of the traps, and $c$ is the ratio between the density of states of the traps and that of the band. The first of Eq. (1) is the transport equation describing the drift motion of the band electrons under the influence of the field, while (2) expresses the power balance of the band electrons: specifically, the $J F$ product at the left hand side provides the power per unit volume injected by the field: such a power is absorbed in part by the phonons (first term at the right hand side), while the other part determines the time change of the band population (second term). Finally, the second of (1) relates $n_{B} / n$, that is, the fraction of the electrons that belong to the band, to the electrons' temperature.

The three model equations $(1,2)$ involve four unknowns, namely, $J, F, n_{B}$, and $T_{e}$. In a steady-state condition, the equations reduce to a single, algebraic relation involving $F$ and $J$ only. However, the use of the $F, J$ variables is awkward, and it is found that the adoption of auxiliary variables is more suitable because it provides expressions that are easily managed analytically without introducing approximations. This is true also for the time-dependent 
TABLE I

PARAMETERS, SYMBOLS, AND AUXILIARY UNKNOWNS,

\begin{tabular}{|c|c|c|c|}
\hline Name or definition & Symbol & Value & Units \\
\hline Room temperature & $T_{0}$ & 300 & $\mathrm{~K}$ \\
\hline Boltzmann constant & $k$ & $1.38 \times 10^{-23}$ & $\mathrm{~J} / \mathrm{K}$ \\
\hline Total electron conc. & $n$ & $1 \times 10^{20}$ & $\mathrm{~cm}^{-3}$ \\
\hline Energy gap & $\Delta E$ & 360 & $\mathrm{meV}$ \\
\hline$\Delta E /\left(k T_{0}\right)$ & $a$ & 13.93 & - \\
\hline Density-of-states ratio & $c$ & $2.5 \times 10^{-4}$ & - \\
\hline $1+c \exp (a)$ & $v^{\mathrm{eq}}$ & 281.4 & - \\
\hline $1+c$ & $v_{m}$ & 1.00025 & - \\
\hline Device length & $L$ & 40 & $\mathrm{~nm}$ \\
\hline Device cross-section & $A$ & $40 \times 40$ & $\mathrm{~nm}^{2}$ \\
\hline Electric field & $F$ & - & $\mathrm{V} \mathrm{m}^{-1}$ \\
\hline Voltage, $L F$ & $\bar{V}$ & - & $\mathrm{V}$ \\
\hline Current density & $J$ & - & $\mathrm{A} \mathrm{m}^{-2}$ \\
\hline Current, $A J$ & $I$ & - & $\mathrm{A}$ \\
\hline- & $L / A$ & $2.5 \times 10^{-2}$ & $\mathrm{~nm}^{-1}$ \\
\hline$q \mu n F_{0}$ & $J_{0}$ & 4.137 & $\mathrm{MA} / \mathrm{cm}^{2}$ \\
\hline$k T_{0} /\left(q \mu \tau_{R}\right)$ & $F_{0}^{2}$ & $1.002 \times 10^{10}$ & $\mathrm{~V}^{2} / \mathrm{cm}^{2}$ \\
\hline- & $F_{0} / J_{0}$ & $2.42 \times 10^{-2}$ & $\Omega \mathrm{cm}$ \\
\hline$L F_{0} /\left(A J_{0}\right)$ & $R_{0}$ & 6049 & $\Omega$ \\
\hline$A J_{0}$ & $I_{0}$ & 66.24 & $\mu \mathrm{A}$ \\
\hline$R_{0} I_{0}$ & - & 397.4 & $\mathrm{mV}$ \\
\hline$q \mu n F / J$ & $v$ & - & - \\
\hline$a / \log [(v-1) / c]$ & $H+1$ & 一 & - \\
\hline$R / R_{0}$ & $k_{R}$ & - & 一 \\
\hline$V_{C} /\left(R_{0} I_{0}\right)$ & $k_{C}$ & 一 & - \\
\hline Relaxation time & $\tau_{R}$ & 1 & ps \\
\hline$t /\left(a \tau_{R}\right)$ & $\beta$ & - & - \\
\hline $1 /\left(a \tau_{R}\right)$ & 一 & 71.79 & $\mathrm{GHz}$ \\
\hline
\end{tabular}

analysis. To proceed along this line one defines the normalization parameters, symbols, and auxiliary unknowns listed in Table I.

\section{Dynamics of the Intrinsic Ovonic Switch}

Here the auxiliary variable $v$ is most suited. In fact, $(1,2)$ become

$$
\mathrm{d} v / \mathrm{d} \beta=v^{2}\left[H(v)-\left(I / I_{0}\right)^{2} v\right]=M(I, v) .
$$

When a purely-resistive circuit (like that obtained by removing the capacitance from the circuit of Fig. 1) is added to the bare Ovonic device, the load curve in the $I, v$ variables is obtained from $V=V_{C}-R I$ and $v=$ $V /\left(R_{0} I\right)$, with $R=R_{L}+R_{S}$ and $R_{0}=L F_{0} /\left(A J_{0}\right)$, and reads $I / I_{0}=k_{C} /\left(v+k_{R}\right)$ (the parameters are defined in Table I). The bias point $I_{q}, v_{q}$ is found by coupling the latter with the steady-state case of (3), to find

$$
H(v)=\frac{k_{C}^{2} v}{\left(v+k_{R}\right)^{2}}, \quad v>1 .
$$

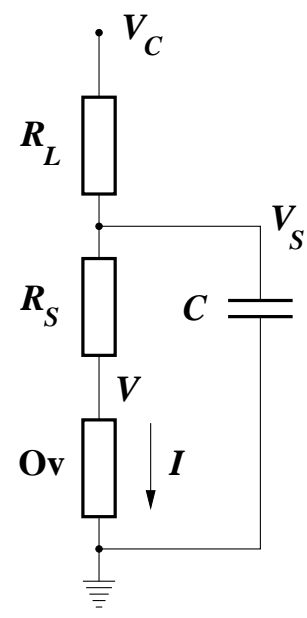

Fig. 1. Circuit obtained by adding the parasitic elements $R_{S}$ and $C$ to the Ovonic device. The other component $R_{L}$ is the input resistance of the measuring apparatus.

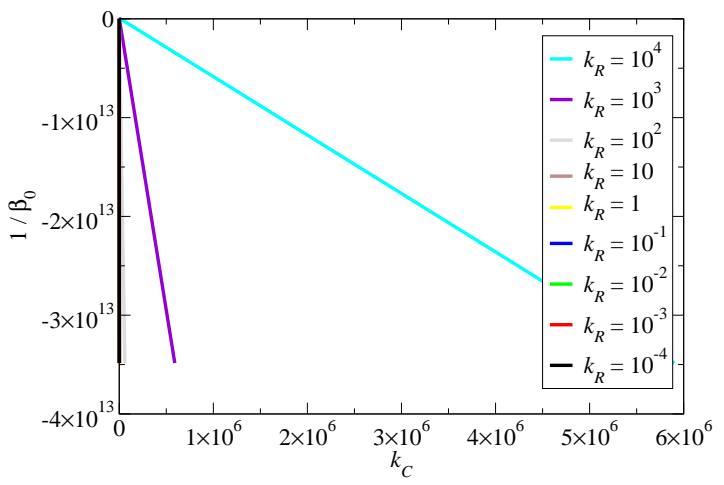

Fig. 2. Calculation of parameter $1 / \beta_{0}$ obtained from the second of (5) for different combinations of $k_{R}=R / R_{0}$ and $k_{C}=$ $V_{C} /\left(R_{0} I_{0}\right)$.

The stability around the bias point is examined by letting $I=I_{q}+\delta I$, to which it corresponds $v=v_{q}+\delta v$. This yields

$$
\frac{\mathrm{d}}{\mathrm{d} \beta} \delta v=\frac{\delta v}{\beta_{0}}, \quad \frac{1}{\beta_{0}}=\left(\frac{\partial M}{\partial I}\right)_{q}\left(\frac{\partial I}{\partial v}\right)_{q}+\left(\frac{\partial M}{\partial v}\right)_{q},
$$

whose solution is $\delta v=\delta v(\beta=0) \exp \left(\beta / \beta_{0}\right)$. The bias point is stable (unstable) if $1 / \beta_{0}<0\left(1 / \beta_{0}>0\right)$. The constant $1 / \beta_{0}$ may have either sign depending on the bias point; its value is found by fixing $V_{C}$ and $R$, then solving (4) to determine the bias point $I_{q}, v_{q}$. One notes that the right hand side of (4) is non negative because $v>1$ and $k_{C} \geq 0$. As a consequence, the solution(s) are possibile only in the range of $v$ where $H \geq 0$. Such a range is $v_{m}<v \leq v_{\text {eq }}$ (the values are given in Table I), with $H$ monotonically decreasing with $v$, and $\lim _{v \rightarrow v_{m}^{+}} H(v)=$ $+\infty, H\left(v^{\text {eq }}\right)=0$. Fig. 2 shows that $\beta_{0}<0$ for the whole range of bias points of practical interest. It follows that if any constant current $I$ is prescribed such that $M \neq 0$ in (3), the auxiliary variable $v$ (proportional to $V$ in this case) exponentially relaxes to yield $M \rightarrow 0$. 


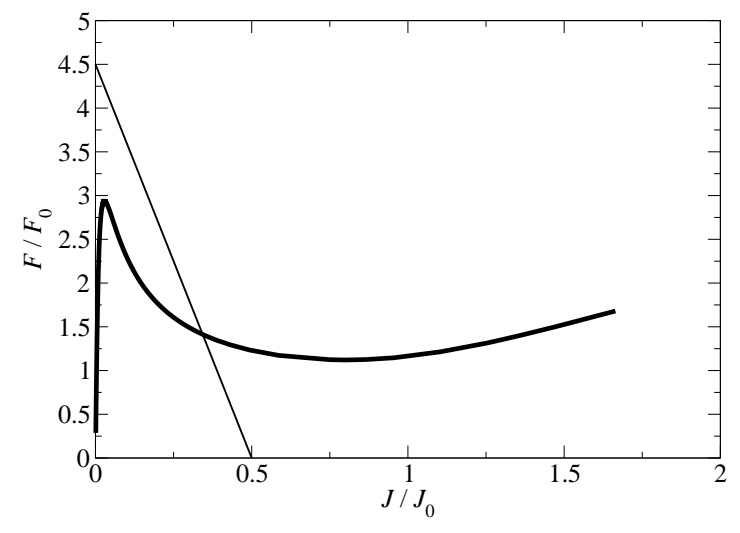

Fig. 3. Normalized field $F / F_{0}$ as a function of the normalized current density $J / J_{0}$. A load curve is shown, such that there is only one intersection with the Ovonic curve, in the negative-slope branch.

\section{Extrinsic Dynamics}

The extrinsic dynamics is dealt with by solving the full circuit of Fig. 1 after letting $\mathrm{d} / \mathrm{d} t=0$ in (2). The values of $R$ and $V_{C}$ are such that there is only one intersection between the Ovonic and load characteristics (Fig. 3, where it is $\left.|\mathrm{d} F / \mathrm{d} J|_{\text {load }}<|\mathrm{d} F / \mathrm{d} J|_{\text {Ov }}\right)$. As shown below, the parameters' values can be chosen in such a way as to make the intersection unstable. Letting $\gamma=t /\left(R_{L} C\right)$ yields

$$
\frac{V_{C}-\left(R_{S}+R_{L}\right) I-V(I)}{R_{S}+\mathrm{d} V / \mathrm{d} I}=\frac{\mathrm{d} I}{\mathrm{~d} \gamma} .
$$

The above equation is separable when $V_{C}=$ const. Letting $q$ still denote the bias point, in the vicinity of it one may replace $\mathrm{d} V / \mathrm{d} I$ with $(\mathrm{d} V / \mathrm{d} I)_{q}=r<0$ and, respectively, $V(I)$ with $V_{q}+r\left(I-I_{q}\right)$, so that (6) becomes

$$
\frac{V_{C}-V_{q}+r I_{q}}{R_{S}+r}=\frac{\mathrm{d} I}{\mathrm{~d} \gamma}+\frac{R_{L}+R_{S}+r}{R_{S}+r} I .
$$

Stability is dictated by the coefficient of $I$ : the bias point is stable when $|r|<R_{S}$ or $|r|>R_{L}+R_{S}$, while it is unstable when $R_{S}<|r|<R_{L}+R_{S}$. Far from the intersection, and considering the $V_{C}=$ const case, the transient analysis must be carried out by solving (6) without approximations. When the parameters are chosen in such a way that $R_{S}<$ $|r|<R_{L}+R_{S}$ holds, an oscillation cycle takes place, like $A \rightarrow B \rightarrow C \rightarrow D \rightarrow A$ of Fig. 4. The $B \rightarrow C$ and $C \rightarrow D$ portions of the cycle are instantaneous due to the neglect of the time derivative in (2). The cycle's period is $t_{\mathrm{cy}}=t_{B}-t_{A}+t_{D}-t_{C}$, where

$$
\frac{t_{B}-t_{A}}{R_{L} C}=\int_{v_{A}}^{v_{B}} S(v) \mathrm{d} v, \quad \frac{t_{D}-t_{C}}{R_{L} C}=\int_{v_{C}}^{v_{D}} S(v) \mathrm{d} v .
$$

The result is shown in Figs. 5 and 6, where the normalized current density $J / J_{0}$ and, respectively, the normalized electric field $F / F_{0}$ are drawn as a function of the normalized time $\left(t-t_{A}\right) /\left(R_{L} C\right)$. Using the data listed in Table I, one finds $\left(t_{B}-t_{A}\right) /\left(R_{L} C\right) \simeq 0.7525$ and $\left(t_{D}-t_{A}\right) /\left(R_{L} C\right) \simeq$ 0.8909. Letting, e.g., $R_{L} \simeq 54.4 \mathrm{k} \Omega, C=1 \mathrm{nF}$, the period

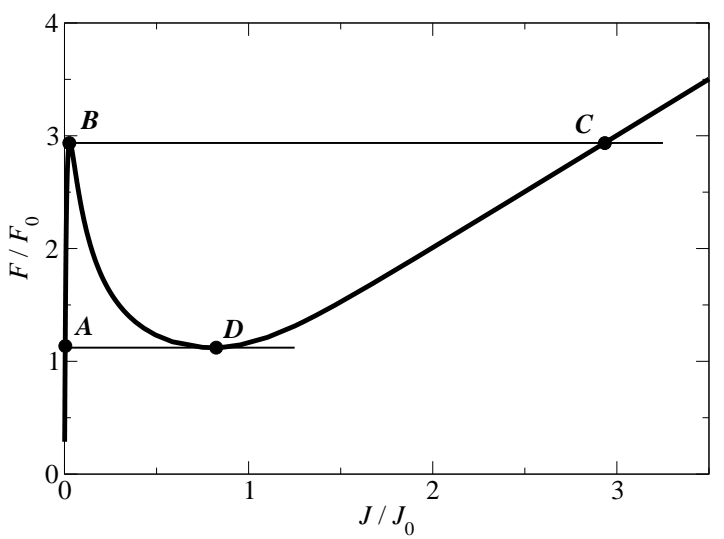

Fig. 4. Extrinsic dynamics. The same curve as in Fig. 3 in a different scale. The points mark the oscillation cycle $A \rightarrow B \rightarrow$ $C \rightarrow D \rightarrow A$, which occurs when the load curve has only one intersect with the Ovonic curve, and the equilibrium point is unstable (see text).

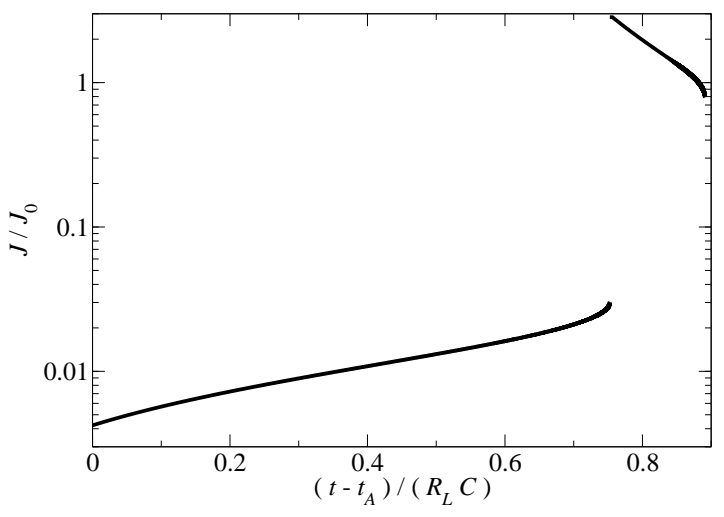

Fig. 5. Extrinsic dynamics. Normalized current density $J / J_{0}$, in a logarithmic scale, as a function of the normalized time $(t-$ $\left.t_{A}\right) /\left(R_{L} C\right)$. The horizontal axis ranges over one period of the cycle.

turns out to be $t_{D}-t_{A} \simeq 0.8909 \times 54.4 \times 10^{3} \times 10^{-9} \simeq 48.5$ $\mu \mathrm{s}$.

\section{Intrinsic and Extrinsic Dynamics}

To examine the behavior of a circuit like that of Fig. 1, when the intrinsic dynamic behavior of the Ovonic device is included in the analysis, one must couple (3), recast in terms of the $I, V$ variables, with the circuit's equation. To this purpose, it is not correct to assume (like in (6)) that $V=V(I)$, because such a relation implies that the static model of the Ovonic device is used. Instead, one must consider $V$ and $I$ as independent variables, so that the intrinsic Ovonic dynamics reads

$$
\begin{gathered}
\frac{\dot{V}}{V}-\frac{\dot{I}}{I}=\frac{Y(V, I)}{I}, \\
Y(V, I)=\frac{V / R_{0}}{a \tau_{R}}\left[H\left(\frac{V}{R_{0} I}\right)-\frac{V I}{L F_{0} A J_{0}}\right] .
\end{gathered}
$$

In this case it is not necessary to resort to an auxiliary variable, because both $I$ and $V$ are the unknowns of a 


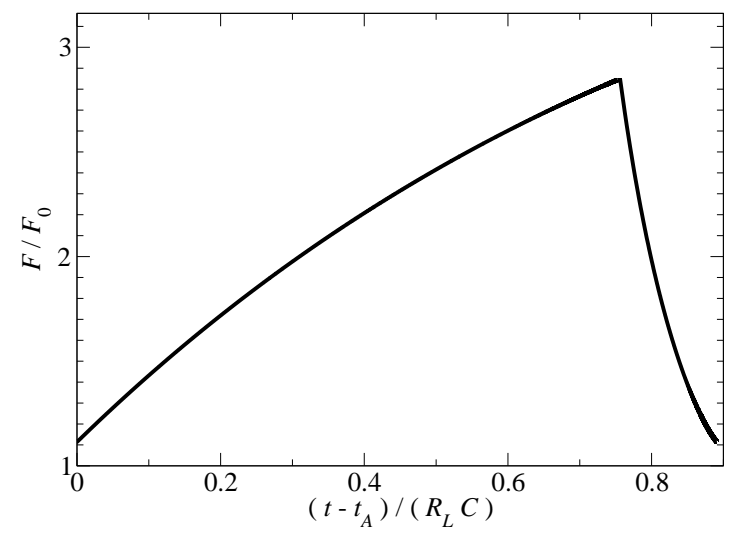

Fig. 6. Extrinsic dynamics. Normalized electric field $F / F_{0}$ as a function of the normalized time $\left(t-t_{A}\right) /\left(R_{L} C\right)$. The horizontal axis ranges over one period of the cycle.

system of equations. In turn, the circuit's equations read

$$
\frac{V_{C}-V_{S}}{R_{L}}=C \frac{\mathrm{d} V_{S}}{\mathrm{~d} t}+I, \quad V_{S}=R_{S} I+V,
$$

equivalent to

$$
\begin{gathered}
\dot{V}+R_{S} \dot{I}=W(V, I), \\
W(V, I)=\left[V_{C}-V-\left(R_{L}+R_{S}\right) I\right] /\left(R_{L} C\right) .
\end{gathered}
$$

Solving (9), (12) for $\dot{V}$ and $\dot{I}$ yields

$$
\dot{V}=V \frac{W+R_{S} Y}{V+R_{S} I}, \quad \dot{I}=\frac{I W-V Y}{V+R_{S} I},
$$

namely, a system of two coupled, non-linear equations of the first order. Such equations provide the global model of the Ovonic device, incorporating the internal relaxation time and the parasitic elements, anticipated in the Introduction. The phase trajectory is obtained from (9), (12) and reads

$$
\mathrm{d} V / \mathrm{d} I=V\left(W+R_{S} Y\right) /(I W-V Y) .
$$

The solution of $(14,15)$ must be tackled by numerical methods. However, a qualitative analysis is feasible when the time constants of the intrinsic and extrinsic dynamics are very different (which is indeed possible if one considers the values reported in Fig. 2). Specifically, with reference to the case where the extrinsic dynamics is unstable, and assuming that $-1 / \beta_{0} \gg R_{L} C$, it is found that the limiting cycle illustrated above becomes an asymptotic condition, to which the Ovonic device is attracted through a much faster exponential relaxation.

\section{Conclusions}

The "intrinsic" and "extrinsic" dynamics of Ovonic switches have been investigated in this paper on the basis of the time-dependent, trap-limited conduction model proposed by the authors. Both dynamics lead to separable, first order equations. The combination of the two can still be reduced to a relatively compact system of equations, giving rise to an asymptotic cycle when, as typical of realistic cases, the time constants are very different.

\section{References}

[1] S. R. Ovshinsky, "Reversible electrical switching phenomena in disordered structures," Phys. Rev. Lett., vol. 21, p. 1450, 1968.

[2] D. Adler, "Switching phenomena in thin films," J. Vac. Sci. Technol., vol. 10, p. 728, 1973.

[3] D. Adler, M. S. Shur, M. Silver, and S. R. Ovshinsky, "Threshold switching in chalcogenide-glass thin films," $J$. Appl. Phys., vol. 51, p. 3289, 1980.

[4] M. Nardone, V. G. Karpov, D. C. S. Jackson, and I. V. Karpov, "A unified model of nucleation switching," Appl. Phys. Lett., vol. 94, p. 103509, 2009.

[5] M. Simon, M. Nardone, V. G. Karpov, and I. V. Karpov, "Conductive path formation in glasses of phase change memory," J. Appl. Phys., vol. 108, p. 064514, 2010.

[6] A. Pirovano, A. Lacaita, A. Benvenuti, F. Pellizzer, and R. Bez, "Electronic switching in phase-change memories," IEEE Trans. Electron Devices, vol. 51, p. 452, 2004.

[7] D. Ielmini and Y. Zhang, "Analytical model for subthreshold conduction and threshold switching in chalcogenide-based memory devices," J. Appl. Phys., no. 102, p. 054517, 2007.

[8] D. Ielmini, "Threshold switching mechanism by highfield energy gain in the hopping transport of chalcogenide glasses," Phys. Rev. B, vol. 78, p. 035308, 2008.

[9] M. Rudan, F. Giovanardi, E. Piccinini, F. Buscemi, R. Brunetti, and C. Jacoboni, "Voltage snapback in amorphous-GST memory devices: Transport model and validation," IEEE Trans. Electron Devices, vol. 58, p. 4361, 2011.

[10] E. Piccinini, A. Cappelli, F. Buscemi, R. Brunetti, D. Ielmini, M. Rudan, and C. Jacoboni, "Hot-carrier trap-limited transport in switching chalcogenides," J. Appl. Phys., vol. 112, p. 083722,2012 .

[11] A. Cappelli, E. Piccinini, F. Xiong, A. Behnam, R. Brunetti, M. Rudan, E. Pop, and C. Jacoboni, "Conductive preferential paths of hot carriers in amorphous phase-change materials," Appl. Phys. Lett., vol. 103, p. 083503, 2013.

[12] S. Raoux and M. Wuttig, Phase Change Materials: Science and Applications. Springer, 2010.

[13] S. Lavizzari, D. Ielmini, D. Sharma, and A. Lacaita, "Transient effects of delay, switching and recovery in phase change memory (PCM) devices," in Proc. International Electron Devices Meeting (IEDM). San Francisco: IEEE, 2008, pp. 1-4.

[14] S. Lavizzari, D. Sharma, and D. Ielmini, "Thresholdswitching delay controlled by $1 / f$ current fluctuations in phase-change memory devices," IEEE Trans. Electron Devices, vol. 57, p. 1047, 2010.

[15] S. Lavizzari, D. Ielmini, and A. Lacaita, "Transient simulation of delay and switching effects in phase-change memories," IEEE Trans. Electron Devices, vol. 57, p. 3257, 2010.

[16] G. W. Burr, R. S. Shenoy, K. Virwani, P. Narayanan, A. Padilla, and B. Kurdi, "Access devices for 3D crosspoint memory," Semicond. Sci. Technol. B, vol. 32, pp. 040 802-1, 2014.

[17] P. E. Schmidt and R. Callarotti, "Theoretical and experimental study of the operation of ovonic switches in the relaxation oscillation mode. i. the charging characteristic during the off state," J. Appl. Phys., vol. 55, p. 3144, 1984.

[18] R. Callarotti and E. Schmidt, "Theoretical and experimental study of the operation of ovonic switches in the relaxation oscillation mode. ii. the discharging characteristic of the equivalent circuit," J. Appl. Phys., vol. 55, p. 3148, 1984.

[19] F. Buscemi, E. Piccinini, A. Cappelli, R. Brunetti, M. Rudan, and C. Jacoboni, "Electrical bistability in amorphous semiconductors: a basic analytical theory," Appl. Phys. Lett., vol. 104, p. 022101, 2014

[20] F. Buscemi, E. Piccinini, R. Brunetti, M. Rudan, and C. Jacoboni, "Time-dependent transport in amorphous semiconductors: Instability in the field-controlled regime," Appl. Phys. Lett., vol. 104, p. 262106, 2014. 